\title{
Surmounting Barriers: The Benefit of Hydrodynamic Interactions
}

\author{
Christoph Lutz, ${ }^{1}$ Michael Reichert, ${ }^{2}$ Holger Stark, ${ }^{2}$ and Clemens Bechinger ${ }^{1}$ \\ 12. Physikalisches Institut, Universität Stuttgart, Pfaffenwaldring 57, D-70550 Stuttgart, Germany \\ ${ }^{2}$ Fachbereich Physik, Universität Konstanz, D-78457 Konstanz, Germany
}

(Dated: October 24, 2018)

\begin{abstract}
We experimentally and theoretically investigate the collective behavior of three colloidal particles that are driven by a constant force along a toroidal trap. Due to hydrodynamic interactions, a characteristic limit cycle is observed. When we additionally apply a periodic sawtooth potential, we find a novel caterpillar-like motional sequence that is dominated by hydrodynamic interactions and promotes the surmounting of potential barriers by the particles.
\end{abstract}

PACS numbers: 82.70.Dd, 67.40.Hf, 83.80.Hj

Hydrodynamic interactions (HI) play an important role whenever two or more particles move in a viscous fluid [1, 2]. Due to their long-range nature, they govern the dynamics of colloidal suspensions, e.g., during self- and collective diffusion [3], sedimentation [4], and aggregation processes [5]. Furthermore, HI can lead to pattern formation of rotating motors [6] with a possible two-dimensional melting transition [7] and they are indispensable for the locomotion of microorganisms [8, 9] or in the transport of fluid by beating cilia [10]. While in all these examples many colloids are involved, the effect of $\mathrm{HI}$ in few-particle systems has been investigated only recently. It has been demonstrated that HI mediate the correlated motion of a pair of colloids trapped in optical tweezers [11] and that they give rise to interesting collective behavior, e.g., periodic or almost periodic motions in time [12] or even transient chaotic dynamics in sedimenting three-particle clusters [13].

In this Letter, we experimentally and theoretically demonstrate how HI lead to a novel motional behavior of a colloidal system comprised of at most three particles. In contrast to the aforementioned examples, where the colloids exhibit $e i$ ther deterministic drift or Brownian diffusion, in the following we concentrate on a non-equilibrium system where both deterministic and stochastic motions are of importance. This work is partially motivated by a recent theoretical analysis of particles driven by a constant tangential force along a toroidal trap [14]. Owing to HI, the particles first go through a transient regime and then enter a characteristic limit cycle. Here, we present the first experimental confirmation of these findings. Our main objective, however, is to investigate experimentally and theoretically how the collective motion of interacting particles changes when a sawtooth potential is added to the constant driving force. Sawtooth potentials are an important component for thermal ratchets studied, e.g., in connection with biological motors [15]. Here, we demonstrate that, due to HI, two-particle clusters exhibit an unexpected caterpillar-like motion which facilitates the surmounting of potential barriers. This motional sequence is largely dominated by hydrodynamic interactions in the system.

Tangential driving forces were exerted on colloidal particles with a single three-dimensional laser tweezer that scans a circle inside our sample cell with the help of computer-controlled mirrors at a frequency $f_{\mathrm{T}}$. In contrast to high scanning speeds $\left(f_{\mathrm{T}}>200 \mathrm{~Hz}\right)$, where the particle motion is entirely diffusive [16], it has been experimentally and theoretically demonstrated that, at intermediate scanning speeds where the particle cannot directly follow the trap, a small periodic force is transferred from the passing optical trap onto the particle [17]. As a result, the particle moves with a mean velocity $v_{0} \propto f_{\mathrm{T}}^{-1}$. For the experimental parameters in our setup $(\lambda=532 \mathrm{~nm}$, $P \approx 200 \mathrm{~mW}, f_{\mathrm{T}}=76 \mathrm{~Hz}$ ), this yields $v_{0} \approx 7 \mu \mathrm{m} / \mathrm{s}$ for a single silica sphere with radius $a=1.5 \mu \mathrm{m}$ immersed in ethanol solution. Since the particle displacement by a single kick from the optical trap is estimated to be only about $0.08 a$, the particle motion is rather smooth. In addition, the focus size slightly changes along the circle which in total leads to a variation of $v_{0}$ smaller than $20 \%$. This means that we can also view $v_{0}$ as the result of a constant driving force $k_{0}=6 \pi \eta a v_{0}$. To avoid wall effects which further complicate the theoretical treatment of $\mathrm{HI}$, the focus of the laser beam was about $40 \mu \mathrm{m}$ above the bottom plate of our sample cell. The particles were illuminated with a white light source and imaged to a CCD camera, which was connected to a PC where images were compressed and stored. Particle trajectories were obtained offline by a particle tracking algorithm [18]. To enhance the electrostatic coupling between the charged spheres, no salt was added to the suspension.

A sequence of typical snapshots $(\Delta t=4.8 \mathrm{~s})$ of three particles in the periodic limit cycle driven counter-clockwise on a circular ring with $9.86 \mu \mathrm{m}$ radius is shown in Fig. 1 The two-particle cluster at the top [Fig. 1 a)] catches up with a preceding third particle [Fig. 1 b)] until they form a triplet for a short time. Since the middle particle in this triplet is most efficiently screened from the fluid flow, it pushes the frontmost particle ahead so that the two front particles leave the last one behind [Fig.11 (c)]. Due to drag reduction by drafting, this cluster is then again catching up with the single particle [Fig. 11d)]. We also investigated the transition from the unstable particle configuration, where the particles had originally the same distance, into the periodic limit cycle and found very good agreement with theoretical predictions (data not shown) [14]. It should be also mentioned that the limit cycle described above is entirely absent when repeating the experiment close to a surface. We believe that this is a result of the rigid bound- 


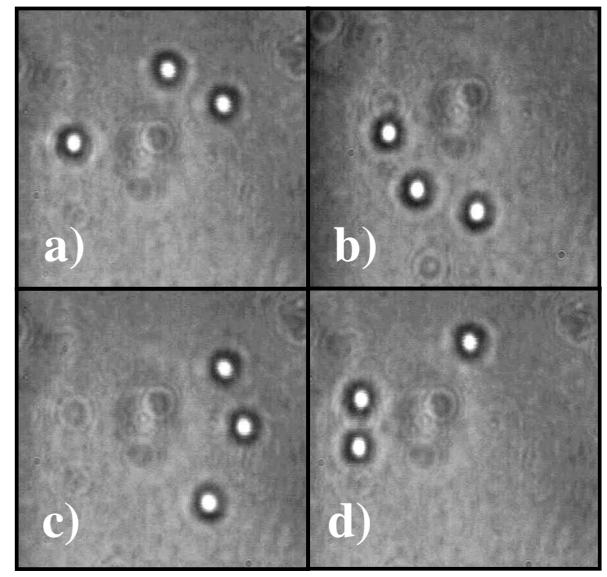

FIG. 1: Snapshots of a video sequence [19] describing the characteristic limit cycle of three colloidal particles (bright) driven along a toroidal trap in counter-clockwise direction. The time difference between the pictures is $4.8 \mathrm{~s}$ each. The optical trap is blocked with optical filters.

ary that alters HI close to surfaces [20].

After having demonstrated hydrodynamic effects in the presence of a constant driving force, we now want to study the cooperative particle motion in the presence of a more complicated force profile. In addition to the constant driving force $k_{0}>0$, we apply a sawtooth potential $V_{\mathrm{s}}(x)$ with period $L$, where $x$ is the arc-length coordinate along the circumference of the trap. In the first segment of length $L_{1}, V_{\mathrm{s}}(x)$ exerts an additional force $k_{\mathrm{s} 1}>0$ on the particle, thus enhancing the drift motion, whereas in the second segment of length $L_{2}=L-L_{1}$, the force $-k_{\mathrm{s} 2}<0$ counteracts $k_{0}$. As a result, the particle is moving in a tilted sawtooth potential $V(x)$, as illustrated in the inset of Fig. 2 a). The single-particle motion is described by the one-dimensional Smoluchowski equation from which the probability distribution $p(x, t)$ for finding the particle at position $x$ in the tilted sawtooth potential is calculated. Since the particle moves in a circle, $p(x, t)$ is stationary. After solving the Smoluchowski equation on the two segments using appropriate matching conditions at $x=0, L$ and $x=L_{1}$, we calculate the constant probability current $j$ and finally arrive at the mean particle velocity $\langle v\rangle=j L[21]$ :

$$
\begin{aligned}
\frac{\langle v\rangle}{v_{0}}=[1+ & \frac{q^{2}}{(1-q)[1-(1-q) \delta]} \\
& \left.\times\left(\delta-\frac{1}{C} \frac{1-e^{-(1-q) \delta C}}{(1-q)[1-(1-q) \delta]}\right)\right]^{-1} .
\end{aligned}
$$

Here, $v_{0}=k_{0} /(6 \pi \eta a)$ is the particle velocity without sawtooth potential, and $\delta=L_{2} / L$ describes the asymmetry of the potential. $C=k_{0} L /\left(k_{\mathrm{B}} T\right)$ is the energy dissipated on the length $L$ relative to the thermal energy $k_{\mathrm{B}} T$. It is linked to the conventional Peclet number $\mathrm{Pe}=C a / L$. Since $C \gg 1$ in our case, one might naively expect a purely deterministic motion. In general, however, the type of motion depends on the value $q=k_{\mathrm{s} 2} / k_{0}$ which serves as a measure for the amplitude of the

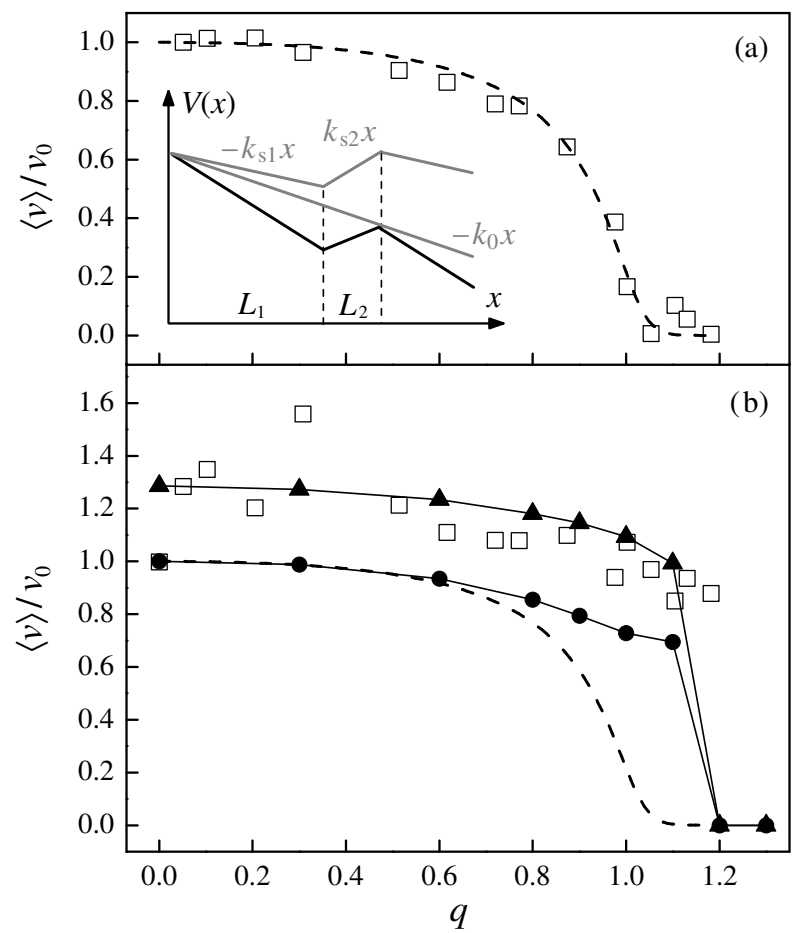

FIG. 2: (a) Normalized single-particle velocities as obtained from experiments $(\square)$ and from the analytical expression 11 (- - -). Inset: Schematic representation of the tilted sawtooth potential. (b) Corresponding two-particle-cluster velocities from experiments $(\square)$ and numerical simulations including $\mathrm{HI}(\boldsymbol{\Delta})$ and without $\mathrm{HI}(\mathbf{O})$. For comparison, the dashed curve of (a) is replotted.

sawtooth potential. For $q<1$, the motion is indeed purely deterministic and determined by the first term in the second line of Eq. (11. At $q=1$, the net force acting on the particle in segment $L_{2}$ vanishes $\left(k_{0}-k_{\mathrm{s} 2}=0\right)$, and the colloid moves entirely stochastically until it resumes the drift motion in segment $L_{1}$. At $q>1$, it even experiences a potential barrier that gives rise to a stick-slip motion. Fig.2] a) (dashed line) shows the result of Eq. (1) as a function of $q$ for the set of experimental parameters specified below. Note that, in the limit $C \rightarrow \infty$, the Boltzmann tail at $q>1$ vanishes and $\langle v\rangle$ becomes zero at $q=1$.

To realize the situation described above experimentally, we weakly modulated $(\leq \pm 12 \%)$ the intensity of the scanning optical tweezer. This was achieved with an electro-opticalmodulator controlled by a function generator that was synchronized with the scanning motion of the laser focus. Before discussing hydrodynamic coupling of particles in such a situation, let us briefly demonstrate that our experimental approach allows to simultaneously apply a constant drift force and a quasi-static periodic potential to the colloids. The upper inset of Fig. 3 shows the position dependent velocity $v(x)$ of a single particle (averaged over $200 \mathrm{~ms}$ each) determined from its trajectory. From this, we calculate the position dependent force $k(x)=6 \pi \eta a v(x)$ and obtain the energy dissipated by the particle, $W_{\text {diss }}(x)=-\int_{0}^{x} k\left(x^{\prime}\right) d x^{\prime}$ (Fig. 3), which 


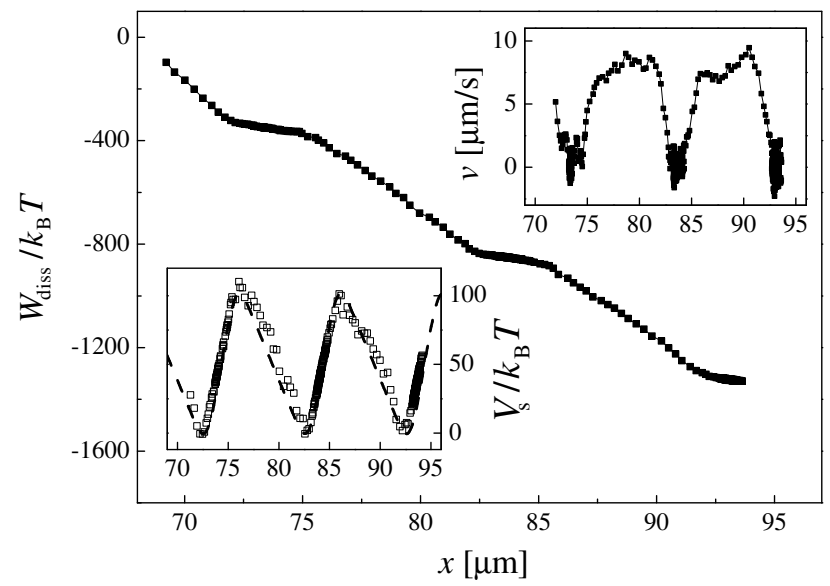

FIG. 3: Experimentally determined dissipated energy of a particle in a sawtooth potential with a constant driving force. Upper inset: position-dependent velocity of a single particle. Lower inset: sawtooth potential as obtained from the dissipated energy $(\square)$ and as calculated from the intensity variation along the contour of the toroidal trap (- - ).

is essentially identical to the tilted sawtooth potential $V(x)$ [22]. After subtraction of the linear contribution $k_{0} x$ corresponding to the mean particle velocity $v_{0}$ measured without intensity modulation, we finally obtain the spatially periodic potential acting on the particles. The open symbols of the lower inset in Fig. 3 show the corresponding sawtooth potential. On the other hand, taking into account the size of the laser focus and that of the particles (both effects lead to some rounding of the edges in the potential), we can calculate from the sawtooth-shaped modulation of the laser intensity the effective potential acting on the particles [21]. It is shown as dashed line and demonstrates the good agreement with our measurements. The amplitude of the potential corresponds to an intensity modulation amplitude of $\Delta P \approx 20 \mathrm{~mW}$ that is consistent with the measured intensity variation.

First, we experimentally determined the normalized mean particle velocity $\langle v\rangle / v_{0}$ of a single particle [see Eq. (1)] as a function of the amplitude of the sawtooth potential, the latter being proportional to $q$ (with the proportionality constant as fitting parameter). The results are plotted in Fig. (2 a) as open symbols and were obtained for the following experimental parameters: $v_{0}=7.24 \mu \mathrm{m} / \mathrm{s}$ and $\delta=L_{2} / L \approx 2.7 \mu \mathrm{m} / 10.3 \mu \mathrm{m}$ which yields $k_{0}=245 \cdot 10^{-15} \mathrm{~N}$ and $C \approx 610$. The dashed curve in Fig. 22 a) is the result of a least-mean-square fit to Eq. (1) that shows excellent agreement with the data when $L_{2}$ as a free fitting parameter assumes the value $L_{2}=1 \mu \mathrm{m}$ and thus $\delta=0.1$. We attribute the deviation from the experimental $L_{2}$ to the difference between the experimentally realized and the perfect sawtooth potential as mentioned above.

Next, we investigated the case where three particles were driven along the sawtooth potential. Similar to the case $V_{\mathrm{s}}(x) \equiv 0$, the particles change their relative distances as a function of time (for video sequences see [19]). In contrast to Fig. 1 however, we do not observe the same periodic limit

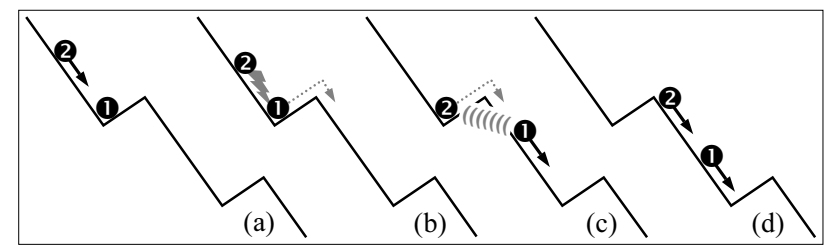

FIG. 4: Sketch of the motional sequence of a cluster comprised of two particles. Due to HI and electrostatic interactions, a caterpillarlike motion is observed which facilitates the surmounting of potential barriers.

cycle but a novel type of collective motion induced by the sawtooth landscape. Figure 2 b) shows the average velocity of two-particle clusters in a tilted sawtooth potential as determined from experiments (open symbols) and from Browniandynamics simulations (solid triangles) with the above parameters. In our image analysis, we defined a cluster as a pair of particles with center-to-center distance $\leq 5 a$, corresponding to arc-length distance $\leq 0.7 \mathrm{~L}$. Obviously, the two-particle velocity is larger than the corresponding one-particle velocity which is again a result of the reduced hydrodynamic friction. What is actually surprising, is the fact that even when the single-particle velocity drops to zero around $q \geq 1$ (because the particle becomes trapped in a potential well), the cluster velocity varies only by about $20 \%$ in that $q$-range. This clearly demonstrates that the surmounting of potential barriers is largely facilitated for particle clusters compared to a single colloid. The reason for this behavior is due to HI that lead to an interesting, caterpillar-like motion of the clusters as described in the following. Assume particle "2" [Fig. [4a)] drifts into a potential well which is already occupied by particle "1". Due to a combined effect of electrostatic repulsion and HI, particle " 2 " will push particle " 1 " over the barrier [Fig. 4b)]. This in turn causes a hydrodynamic drag that particle "1" exerts onto particle "2" [(Fig. 4 ( $\mathrm{c})]$ which pulls particle " 2 " across the barrier [Fig. 4 (4)]. The motional sequence just described is reminiscent to that of a caterpillar which first stretches out, adheres at the front, and then pulls the tail towards the head. Once such a mode is initiated (e.g., by a thermally induced process), the outlined motion may last over several periods $L$ until the particles become trapped in separate potential minima due to thermal fluctuations of their distance. Then, the motion stops until the situation described in Fig. 4 (a) is initiated by a spontaneous hop of particle "2" into the potential well occupied by particle "1".

This motional pattern of two-particle clusters was also observed in our Brownian-dynamics simulations. The electrostatic inter-particle repulsion is described by a screened Coulomb potential [23] $V_{\text {rep }}(r)=\left[Z e^{\kappa a} /(1+\right.$ $\kappa a)]^{2} \lambda_{\mathrm{B}} k_{\mathrm{B}} T e^{-\kappa r} / r$ with Debye length $\kappa^{-1} \approx 300 \mathrm{~nm}$, where the effective particle charge is adjusted to $Z \approx 8000$ (taking $\lambda_{\mathrm{B}} \approx 0.7 \mathrm{~nm}$ for the Bjerrum length in water) so that the center-to-center distance between the particles does not go below about $3 a$ as observed in the experiment. The Browniandynamics simulations including HI were carried out using a 
predictor-corrector-type integration scheme which is first order in the time step $\Delta t[21]$. For particle separations $r \geq 3 a$, the mobilities describing the $\mathrm{HI}$ and thus the mutual coupling of all particles can be well approximated by the Rotne-Prager tensor [2], which is the far-field expansion up to order $1 / r^{3}$ (for details, see Ref. [21]).

To demonstrate the crucial role of $\mathrm{HI}$ for the enhanced cluster motion, we performed numerical simulations without HI. The mean particle velocity, defined as the average over all particles and times, coincides with the single-particle curve in Fig. 2 when plotted as a function of $q$. This is clear since, due to the average over all particles, the electrostatic forces cancel each other $($ actio $=$ reactio) and thus, on average, the particles move independently. The mean velocities of the particle clusters, however, deviate from the single-particle velocities, as illustrated in Fig. 2]b) (solid circles). Nevertheless, as expected, the average cluster velocities are always below the corresponding curve where HI are included. For $q \geq 1$, the two-particle-cluster motion without HI consists of singular events where particle " 2 " moves down the seceding flank of the tilted sawtooth potential and pushes particle " 1 " over the potential barrier [Fig.4 (a,b)]. In contrast to the caterpillar-like behavior, the collective motion stops here since the drag due to HI is missing. Then, another thermal activation is necessary to induce the sequence again [19].

Finally, we point out that the short-range electrostatic repulsion between the particles is only of minor importance for the collective, caterpillar-like motion as described above. It is rather the long-ranged $\mathrm{HI}$ that dominate the force which pushes the front particle in Fig. 4lb) over the barrier (note that HI decay asymptotically as $1 / r$ whereas the dominant term in the repulsive electrostatic force is exponentially damped, $F_{\text {rep }}(r)=-\nabla_{r} V_{\text {rep }}(r) \propto e^{-r / \lambda_{\mathrm{D}}} / r$ with $\left.\lambda_{\mathrm{D}} \ll a\right)$. The caterpillar-like motion was also obtained in simulations where the strength of the electrostatic interaction potential was reduced by a factor of 50 so that the minimum distance to contact of the particles was $0.1 a[21]$.

The present study demonstrates that HI strongly dominate the motional behavior of driven colloidal particles. In the presence of a constant driving force, we experimentally confirmed the recently predicted characteristic limit cycle. If in addition a static periodic potential is applied along the toroidal trap, we find that colloidal clusters perform a caterpillar-like motion which facilitates the surmounting of large potential barriers. This novel type of motion which is predominantly triggered by HI is also confirmed by numerical simulations. In addition to a better microscopic understanding of $\mathrm{HI}$ in few-particle systems, our results are also of relevance for the motion of interacting particles in thermal ratchets, which are considered as models for biological motors. Indeed, recent invivo experiments demonstrate that the speed of coupled motor proteins is increased compared to the speed of a single motor [24].

We would like to thank V. Blickle for helpful discussions. This work was supported by the Deutsche Forschungsgemeinschaft through SFB Transregio 6 and Grant No. Sta 352/5-1.
[1] J. Happel and H. Brenner, Low Reynolds Number Hydrodynamics (Noordhoff, Leyden, 1973).

[2] J.K.G. Dhont, An Introduction to Dynamics of Colloids (Elsevier, Amsterdam, 1996).

[3] P.N. Pusey, in Liquids, Freezing, and Glass Transition, Proceedings of the Les Houches Summer School of Theoretical Physics 1989, Part II, edited by J.P. Hansen, D. Levesque, and J. ZinnJustin (North-Holland, Amsterdam, 1991), p. 763; G. Nägele, Phys. Rep. 272, 215 (1996).

[4] P.N. Segrè, E. Herbolzheimer, and P.M. Chaikin, Phys. Rev. Lett. 79, 2574 (1997); M.P. Brenner, Phys. Fluids 11, 754 (1999); A.J.C. Ladd, Phys. Rev. Lett. 88, 048301 (2002); S.Y. Tee, P.J. Mucha, L. Cipelletti, S. Manley, M.P. Brenner, P.N. Segrè, and D.A. Weitz, Phys. Rev. Lett. 89, 054501 (2002); J.T. Padding and A.A. Louis, Phys. Rev. Lett. 93, 220601 (2004).

[5] H. Tanaka and T. Araki, Phys. Rev. Lett. 85, 1338 (2000).

[6] B.A. Grzybowski, H.A. Stone, and G.M. Whitesides, Nature 405, 1033 (2000).

[7] P. Lenz, J.-F. Joanny, F. Jülicher, and J. Prost, Phys. Rev. Lett. 91, 108104 (2003).

[8] E.M. Purcell, Am. J. Phys. 45, 3 (1977).

[9] H.C. Berg, Nature 245, 380 (1973); M.J. Kim, J.C. Bird, A.J. Van Parys, K.S. Breuer, and T.R. Powers, Proc. Natl. Acad. Sci. USA 100, 15481 (2003); M. Reichert and H. Stark, Eur. Phys. J. E 17, 493 (2005).

[10] S. Gueron and K. Levit-Gurevich, Proc. Natl. Acad. Sci. USA 96, 12240 (1999).

[11] J.-C. Meiners and S.R. Quake, Phys. Rev. Lett. 82, 2211 (1999); S. Henderson, S. Mitchell, and P. Bartlett, Phys. Rev. E 64, 061403 (2001); M. Reichert and H. Stark, Phys. Rev. E 69, 031407 (2004).

[12] R.E. Caflisch, C. Lim, J.H.C. Luke, and A.S. Sangani, Phys. Fluids 31, 3175 (1988); I.K. Snook, K.M. Briggs, and E.R. Smith, Physica A 240, 547 (1997).

[13] I.M. Jánosi, T. Tel, D.E. Wolf, and J.A.C. Gallas, Phys. Rev. E 56, 2858 (1997).

[14] M. Reichert and H. Stark, J. Phys.: Condens. Matter 16, S4085 (2004).

[15] F. Jülicher, A. Ajdari, and J. Prost, Rev. Mod. Phys. 69, 1269 (1997).

[16] C. Lutz, M. Kollmann, and C. Bechinger, Phys. Rev. Lett. 93, 026001 (2004).

[17] L.P. Faucheux, G. Stolovitzky, and A. Libchaber, Phys. Rev. E 51, 5239 (1995).

[18] J. Baumgartl and C. Bechinger, Europhys. Lett. 71, 487 (2005).

[19] for videos see www.physik.uni-stuttgart.de/institute/pi/2/Bechinger/research/more/caterpillar/index.shtml

[20] H. Diamant, B. Cui, B. Lin, and S.A. Rice, J. Phys.: Condens. Matter 17, S2787 (2005).

[21] M. Reichert, C. Lutz, C. Bechinger, and H. Stark, in preparation.

[22] $W_{\text {diss }}$ also contains small contributions from the stochastic motion of the particles that can be neglected relative to its absolute value.

[23] B.V. Derjaguin and L. Landau, Acta Physicochim. (USSR) 14, 633 (1941); E.J. Verwey and J.T.G. Overbeek, Theory of the Stability of Lyophobic Colloids (Elsevier, Amsterdam, 1948).

[24] C. Kural, H. Kim, S. Syed. G. Goshima, V. I. Gelfand, and P. R. Selvin, Science 308, 1469 (2005). 